\title{
Molecular spintronics: spin-dependent electron transport in molecular wires
}

\author{
Eldon G. Emberly ${ }^{\mathrm{a}, *}$, George Kirczenow ${ }^{\mathrm{b}}$ \\ ${ }^{a}$ NEC Research Institute, 4 Independence Way, Princeton, NJ 08540, USA \\ ${ }^{\mathrm{b}}$ Department of Physics, Simon Fraser University, Burnaby, BC, Canada, V5A IS6
}

Received 21 September 2001

\begin{abstract}
We present a theoretical study of spin-dependent transport through molecular wires bridging ferromagnetic metal nanocontacts. We extend to magnetic systems a recently proposed model that provides a quantitative explanation of the conductance measurements of Reed et al. [Science 278 (1997) 252] on Au break-junctions bridged by self-assembled molecular monolayers (SAMs) of 1,4-benzene-dithiolate (BDT) molecules. Based on our calculations, we predict that spin-valve behavior should be observable in nickel break-junctions bridged by SAMs formed from BDT. We also consider spin transport in systems consisting of a clean ferromagnetic nickel STM tip and SAMs of benzene-thiol molecules on gold and nickel substrates. We find that spin-valve behavior should be possible for the Ni substrate. For the case where the substrate is gold, we show that it should be possible to inject a highly spin-polarized current into the substrate. (ㄷ 2002 Elsevier Science B.V. All rights reserved.
\end{abstract}

\section{Introduction}

Over the past few years, molecular wires formed by bridging pairs of metallic contacts with organic molecules, have been shown to display a remarkable range of electron transport phenomena [1-8] Some wires display rectification [3], others yield negative differential resistance [7] and/or transistor action [8], and still others involve molecules that are known to switch conformations and exhibit switching behavior in their transport characteristics [4]. As this special issue on electron transport will attest, understanding the measured experi-

\footnotetext{
${ }^{*}$ Corresponding author.
}

mental data is a great challenge that requires bringing together principles from both chemistry and physics. A wide variety of theoretical approaches have arisen over the past few years for the modeling of molecular wire systems. These range from self-consistent calculations [9-17] to semi-empirical methods [18-22]. Molecular wires have great promise from a pure science perspective and also potentially for applications. Hence both experimental and theoretical research in this field continues to attract increasing interest.

Another area of research that has also seen great growth over the past few years is spintronics, a branch of electronics that employs the electron's spin degree of freedom as well as its charge to store, process and transmit information [23-25]. 
Important spintronic phenomena include giant magnetoresistance, spin-valve behavior and injection of spin-polarized currents from ferromagnetic materials to paramagnetic materials [26-37]. The prototypical spin-valve system consists of two ferromagnetic leads separated by a paramagnetic coupling layer. Transport is measured between the two ferromagnetic layers under an applied voltage. The system functions as a spin-valve when it exhibits a difference in its electrical conductance between configurations in which the magnetizations of the ferromagnetic layers are aligned parallel and anti-parallel. Different materials can display very different spintronic properties. For example, injection of spin currents from metallic ferromagnets into non-magnetic metals is relatively straight forward and was first demonstrated experimentally more than a decade ago [28]. Since then it has found important practical applications in information storage technology [23]. However, attempts at demonstrating efficient spin injection from ferromagnetic metals into semiconductors through solid state interfaces have encountered fundamental obstacles [33] and work is underway to determine whether these may be overcome through the use of potential barriers and/or interfaces that obey certain selection rules [33-37].

Much less is known at the present time about spin transport in molecules. There have been a few studies of spintronic systems involving carbon nanotubes [38,39]. There has also been theoretical work looking at transport through paramagnetic ions bridging two electrodes in the presence of a magnetic field [40]. However, to our knowledge whether functioning self-assembled nanoscale spintronic devices can be made out of organic molecules and magnetic nanocontacts has not as yet been explored experimentally or theoretically. In this paper, we show that spin-dependent transport phenomena should be readily observable in such molecular wire systems using currently available experimental techniques. We consider two classes of systems: break-junctions made from ferromagnetic nickel bridged by self-assembled monolayers of organic molecules, and STM setups consisting of a clean single-crystal nickel tip and an SAM on a gold or nickel (100) substrate. Schematics of these systems are shown in Fig. 1.

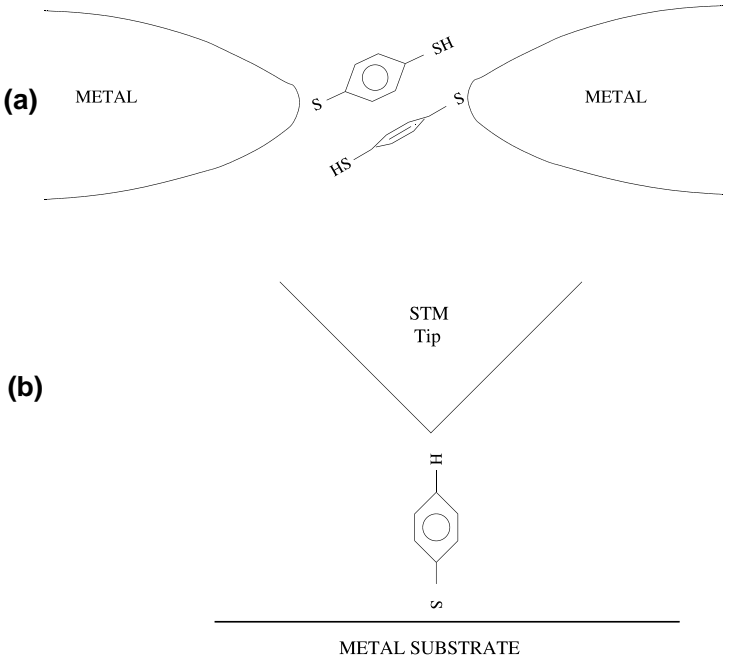

Fig. 1. Schematic of two molecular wire configurations: (a) metal break-junction bridged by self-assembled monolayers of organic molecules; (b) clean STM tip approaches a self-assembled molecular monolayer of organic molecules on a metal substrate.

Our calculations suggest that spin-valve effects and spin injection should be possible in these molecular wire based systems.

As a reference point for the present calculations, we briefly revisit a recently proposed model that has been instrumental in achieving a quantitative understanding [22] of current flow in self-assembled molecular wires bridging a non-magnetic break-junction. In this model, it is assumed that current flows not through a single molecule connecting the two tips of the metal break-junction as in previous theories, but through overlapping molecules, with each molecule chemically bonded to only one metal tip. This model together with a semi-empirical method for the solution of the transport problem is able to quantitatively reproduce [22] the measured conductance data of Reed et al. [1] for gold break junctions bridged by benzene-dithiol molecules. Because it is not computeintensive, a semi-empirical theoretical approach allows us to calculate the transport properties of an ensemble of possible orientations of the overlapping molecules and to include the effects of positional disorder. Thus we are able to calculate transport properties averaged over configurations. 
We feel that the success of this work has demonstrated that semi-empirical methods are useful as a tool for evaluating and estimating effects that are not easily accessible to ab-initio techniques.

This paper is organized as follows: in Section 2, we give a brief summary of a method for calculating the transport characteristics of a molecular wire. The current is evaluated via Landauer theory and the scattering problem is solved using a semiempirical tight-binding form of Schrödinger's equation. Section 3 contains a summary of the model that we have proposed for understanding electrical conduction in non-magnetic metal breakjunctions bridged by SAM's [22], and also representative results of calculations of the differential conductance of non-magnetic systems that are in quantitative agreement with experiment [1]. These results provide a useful benchmark for comparison with our calculations for magnetic systems that follow. Our spin-dependent transport results are presented in Sections 4 and 5. In Section 4, we consider molecular wires bridging magnetic break junctions. We predict that spin-valve behavior should be experimentally detectable in breakjunction measurements on SAM's, where the break junction is made from ferromagnetic nickel. Section 5 details our results for systems that include magnetic STM tips. We show that spin-injection and spin-valve effects should be observable in the systems that we consider. Finally, in Section 6 we present some conclusions that may be drawn from this work.

\section{A method for evaluating the electrical current in molecular wires}

In this section, we briefly review a method for calculating the conductance properties of a molecular wire system. We consider a molecular wire to consist of a pair of electrodes which act as a source and drain for electrons, that are connected by a molecule or molecules. A bias is applied to the electrodes and an electric current flows through the system. A standard method for evaluating the current in such a system is Landauer theory [41]. It relates the electric current flowing through the molecular conductor to the probability for a single electron to scatter from one electrode to the other. For a two-terminal system, the current as a function of the applied bias $V$ can be calculated from the following equation

$I(V)=\frac{e^{2}}{h} \int \mathrm{d} E T(E, V)\left[F\left(E, \mu_{\mathrm{S}}\right)-F\left(E, \mu_{\mathrm{D}}\right)\right]$,

where $F(E, \mu)$ is the equilibrium Fermi distribution and $\mu_{\mathrm{S}, \mathrm{D}}=E_{\mathrm{F}} \pm \mathrm{eV} / 2$ are the electro-chemical potentials of the electrodes in terms of the common Fermi energy $E_{\mathrm{F}}$ of the source (S) and drain (D) electrode, respectively. The transmission probability $T(E, V)$ is the sum of the transmission probabilities for both spin up and spin down electrons to scatter through the system at energy $E$. For a thorough review of Landauer theory see [42].

A variety of methods exist for solving the scattering problem to determine the transmission probability. These range from self-consistent calculations based on density-functional theory [9-14,16], to simpler semi-empirical tight binding approaches [18-22]. The density-functional methods have the advantage of self-consistently calculating the effects of the electro-static potential that arises in the vicinity of the molecule due to the applied bias $V$ and any charge transfer that may arise between the leads and the molecule. They have the disadvantage of being numerically intensive which restricts the size of the problems to which they can be applied at present. They constitute the present state-of-the-art, but nevertheless they rely on approximations whose reliability for transport calculations is unknown when the system departs significantly from equilibrium, a case that is particularly important for molecular wire systems. Semi-empirical approaches do not include the effects of the electro-static field self-consistently. Many body effects are included implicitly to a limited extent in the parameters, and the electrostatic field may be added as an extra potential which can be used to approximate the true field. Because of the speed with which calculations using semi-empirical methods can be carried out, they have the advantage of being able to study much larger systems, including also the various changeable degrees of freedom that influence transport in these systems. They have been shown to produce quantitatively similar results to density functional 
calculations in the linear voltage regime for systems where the effects of the self-consistent potential are less significant $[20,43,44]$. Thus semiempirical approaches should be considered as a valuable tool in that they are able to provide an efficient method by which to evaluate the importance of various factors which may contribute to the transport properties of the molecular wire of interest. With the important factors determined, more sophisticated approaches can then be used to gain more quantitative understanding. What follows is a summary of the semi-empirical tightbinding approach that we use in this work to solve the scattering problem.

We calculate the transmission probability by explicitly calculating the single electron scattering states. Our starting point is Schrödinger's equation

$H|\Psi\rangle=E|\Psi\rangle$,

where $|\Psi\rangle$ is the single-particle scattering state at energy $E$ and $H$ is the Hamiltonian for the entire system. For a two terminal molecular wire, we consider $H$ to have the form

$H=H_{\mathrm{L}}^{0}+H_{\mathrm{M}}^{0}+H_{\mathrm{R}}^{0}+V_{\mathrm{L}}+V_{\mathrm{R}}+V_{\mathrm{ext}}+V_{\mathrm{SCF}}$

where $H_{\mathrm{L}, \mathrm{M}, \mathrm{R}}^{0}$ are the Hamiltonians for the isolated left lead (L), molecule (M) and right lead (R). The molecule is coupled to the leads via $V_{\mathrm{L}, \mathrm{R}}$ for the left and right leads, respectively. $V_{\mathrm{ext}}$ is the external potential due to the applied bias, and $V_{\mathrm{SCF}}$ represents the self-consistent electro-static potential which depends upon the charge distribution in the system. (In what follows, we no longer consider this term, however, a tight-binding method including this term is given in $[13,14]$.) The scattering state $|\Psi\rangle$ can be written as $|\Psi\rangle=\left|\Psi_{\mathrm{L}}\right\rangle+\left|\Psi_{\mathrm{M}}\right\rangle+$ $\left|\Psi_{\mathrm{R}}\right\rangle$, with the following conditions

$$
\begin{aligned}
& \left|\Psi_{\mathrm{L}}\right\rangle=\left|\Phi_{+, \mathrm{L}}^{\alpha}\right\rangle+\sum_{\alpha^{\prime}} r_{\alpha^{\prime}, \alpha}\left|\Phi_{-, \mathrm{L}}^{\alpha^{\prime}}\right\rangle \\
& \left|\Psi_{\mathrm{M}}\right\rangle=\sum_{i} c_{i}\left|\phi_{i}\right\rangle \\
& \left|\Psi_{\mathrm{R}}\right\rangle=\sum_{\alpha^{\prime}} t_{\alpha^{\prime}, \alpha}\left|\Phi_{+, \mathrm{R}}^{\alpha^{\prime}}\right\rangle
\end{aligned}
$$

where $\left|\Phi_{ \pm, \mathrm{L} / \mathrm{R}}^{\alpha^{\prime}}\right\rangle$ is the $\alpha^{\prime}$ th mode at energy $E$ in either the left or right leads, with the \pm denoting whether the state is a rightward or leftward propagating or evanescent mode. The $\alpha$ th mode is always a rightward propagating mode. The $\left|\phi_{i}\right\rangle$ are the bound eigenstates of the isolated molecule. The $r_{\alpha^{\prime}, \alpha}$ and $t_{\alpha^{\prime}, \alpha}$ are the reflection and transmission coefficients, respectively. The total transmission probability for an electron to go from the left lead to the right lead is given by

$T(E, V)=\sum_{\alpha, \alpha^{\prime}} \frac{v_{\alpha^{\prime}}}{v_{\alpha}}\left|t_{\alpha^{\prime}, \alpha}\right|^{2}$,

where the sum over $\alpha$ is over rightward propagating modes in the left lead and the sum over $\alpha^{\prime}$ is over rightward propagating modes in the right lead; all modes at energy $E$. If the system is magnetic, we assume the effects of spin-orbit coupling on transport through the molecular wire to be negligible [45] and solve for $T(E, V)$ for the spin up and spin down electrons separately.

We solve the above problem by representing the scattering states using a linear combination of atomic orbitals (LCAO) (also known as the tightbinding approximation) [48]. This yields a matrix form of Eq. (2)

$\sum_{j} H_{i j} \Psi_{j}=E \sum_{j} S_{i j} \Psi_{j}$

where $H_{i j}$ is the Hamiltonian matrix in the chosen atomic orbital basis. If the basis of atomic orbitals is non-orthogonal then the non-diagonal elements of the overlap matrix $S_{i j}$ must be included; an exact and easily implemented way to take them into account in molecular transport calculations is described in $[49,50]$ Eq. (8) is a linear system which can be solved in a straightforward manner for the unknowns $r_{\alpha^{\prime}, \alpha}, c_{i}$, and $t_{\alpha^{\prime}, \alpha}$. The various propagating and evanescent modes in the leads are determined using standard transfer matrix methods.

We will use this method to explore spin transport in systems that include ferromagnetic leads below. However, we first discuss our model in the simpler context of a non-magnetic system for which it provides a quantitative explanation of available experimental data. This discussion will serve as a theoretical and experimental benchmark that will be helpful in understanding the predictions for magnetic systems that will follow. 


\section{Transport in non-magnetic metal break-junctions bridged by self-assembled monolayers of organic molecules}

In this section, we present a summary of a model [22] which can quantitatively account for the data measured by Reed et al. [1] on a gold break-junction bridged by self-assembled monolayers (SAMs) of 1,4-benzene-dithiolate (BDT). Measurements on this system at room temperature displayed an apparently insulating region for bias values between approximately -0.7 and $0.7 \mathrm{~V}$, nearly symmetric differential conductance in the range from -4 to $+4 \mathrm{~V}$ and some asymmetry at higher bias. The measured conductance was much lower than the quantum $2 e^{2} / h=(1 / 12.9 \mathrm{k} \Omega)$ that sets the conductance scale for single-channel metallic nanowires and also much lower than the calculated conductances of molecular wires in which a single BDT molecule bridges the gap between the two contacts $[10,15,20,21,43]$; the first experimental conductance plateau occurred at a resistance of $22 \mathrm{M} \Omega$.

In the present model, instead of a single molecule chemically bonding to both leads, we consider the situation where each molecule bonds to only one metal tip and the current flows through overlapping molecules from one lead to the other; Fig. 1(a) is a schematic of the model we consider. It has been previously shown theoretically, that having neighboring molecules through which current can flow can have important consequences [51,52]. The present model accounts for the observed low conductance [1] as a result of the weak electrical coupling between the two overlapping molecules even though there is strong coupling between each molecule and the lead to which it is attached. It can also account for the observed reproducibility of the measurements [1] after repeated separation of the two tips: no chemical bonds are formed between the overlapping molecules or between the two leads, hence the SAM's can be pulled apart and brought back together reversibly. Regarding the observed symmetric behavior of the differential conductance [1], we argue that this symmetry arises from the system having the ability to sample all possible energetically favorable configurations of the overlapping molecules. Hence even though a given configuration of the two molecules may be asymmetric, because of thermal fluctuations the mirror configuration will also be sampled. Hence the average over the transport properties of all configurations yields a symmetric result, the small residual asymmetry of the current voltage characteristic in the experiment [1] being due to differences between the atomic structures of the two tips.

Since both tips are densely coated with molecules we assume that molecules bonded to one tip cannot also bond to the other. Because of this, the ends of the molecules that do not bond chemically to a gold surface remain as thiols (SH). For simplicity, we consider the case where the current is carried predominantly through only a single pair of overlapping molecules. We argue that on average the faces of the two benzene rings should be oriented perpendicularly to each other because of electrostatic effects. The rings of carbons in similar molecules have been shown to have negative charge on their sides, whereas the hydrogens all have positive charge [53]. Thus a favorable configuration of two rings which minimizes the electrostatic energy should be one in which they are oriented perpendicularly to each other; this has been found to give rise to herring-bone patterns in molecular dynamics simulations of SAMs of benzene-thiol and similar molecules on gold [53]. Fig. 1(a) shows a possible configuration, where each molecule is bonded to the end of one tip and the molecules orient themselves to avoid steric clashes and minimize the electrostatic forces between them.

We simulate the above system by representing the break-junction by gold clusters to which we attach two BDT molecules. The gold clusters are in the [ 100$]$ direction and consist of $5 \times 5,4 \times 4$, $3 \times 3$, and $2 \times 2$ layers of atoms, forming 54 atom clusters which form the tips of the metal breakjunction. We only include the gold 6 s orbital as we have found that including all of the orbitals $(6 \mathrm{~s}, 6 \mathrm{p}, 5 \mathrm{~d})$ yields quantitatively similar results for the bonding geometries considered here. The BDT molecule consists of a benzene ring with the one and four hydrogens replaced with sulfur atoms. The sulfur atom that is not bonded to a lead remains a thiol. The sulfur atoms that bond to the 
gold are positioned over a fourfold hollow site $2 \AA$ from the ends of the gold clusters [54]. We use the standard extended Hückel parameters [55] to represent the valence orbitals of each atom in the system, and evaluate the necessary site energies, hopping energies and overlaps that make up the Hamiltonian and overlap matrices that go into the solution of Schrödinger's equation, Eq. (2). We attach to this molecular junction, semi-infinite $6 \times 6$ simple cubic multi-mode leads to act as source and drain. Each atom in the lead is simulated using just a single gold-like s orbital. We include disorder by randomly displacing each atom in the gold clusters by a distance consistent with the Debye-Waller factor for gold. For the BDT molecule, we consider random displacements of each atom in the plane of the ring. We do this to approximate the vibrational disorder that will be present in the above systems at room temperature. For the overlapping ring system, we consider one molecule to assume a random tilt angle (between $20^{\circ}$ and $40^{\circ}$ ) with respect to the normal from the gold surface and then orient the second BDT molecule so that its ring is oriented perpendicular to the first, allowing for $20^{\circ}$ fluctuations. We impose steric constraints by demanding that the atoms of the two BDT molecules be separated by distances $\geqslant 3 \AA$. The transmission probability shown below is the average of transmission probabilities from 50 different atomic configurations.

In Fig. 2(a), the average transmission probability is shown as a function of incident electron energy. There are several broad resonances. These resonances are due to highly hybridized molecule/ gold states, with the resonances below $-10 \mathrm{eV}$ being due to the molecular HOMO states and those above $-9 \mathrm{eV}$ arising from the LUMO states. The resonances at $-8.2 \mathrm{eV}$ are due to the LUMO $\pi^{*}$ state which is localized on the benzene ring. The multiple resonances at the LUMO are due not only to vibrational disorder but also to the fact that the wave function overlap between the two molecules results in a splitting of the almost degenerate LUMO levels. In the limit of averaging over an infinite number of configurations these peaks would smear into one broad peak [56].

The solid curve in Fig. 2(b) shows the calculated differential conductance at room temperature for
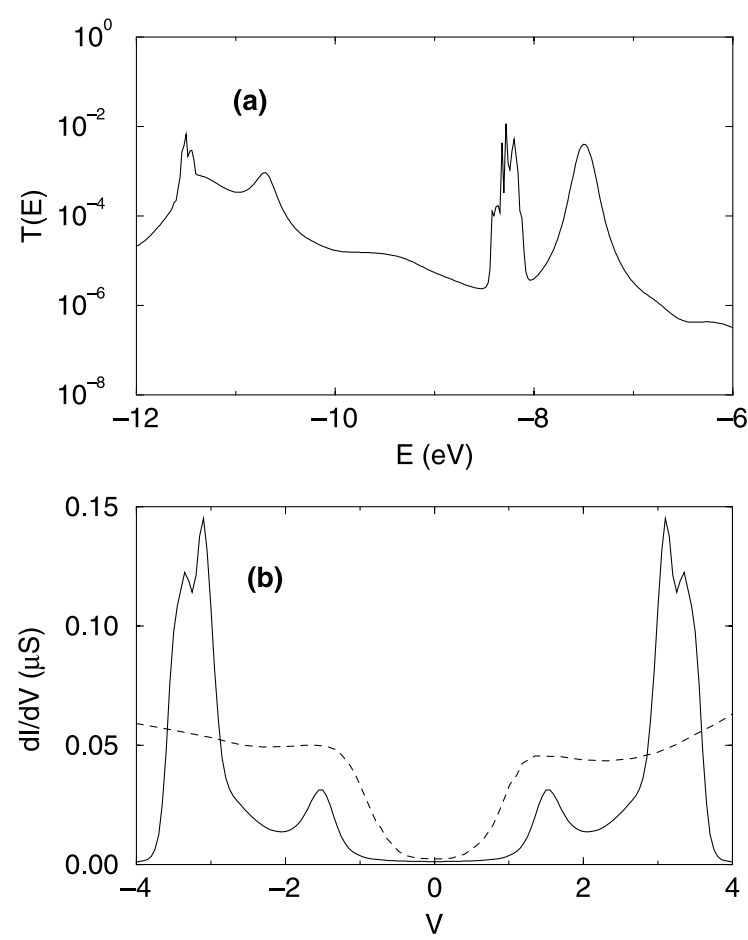

Fig. 2. (a) Calculated average transmission probability at zero applied bias over 50 different atomic configurations for the system shown in Fig. 1(a), where the break-junction is made of gold. (b) Solid line: calculated differential conductance at room temperature using a Fermi energy of the gold leads of $-10 \mathrm{eV}$. Dashed line: experimental differential conductance from [1].

the case of overlapping molecules using a Fermi energy for the gold leads of $-10 \mathrm{eV}$. The first rise in conductance is due to resonant transmission between the HOMO of the molecules. The second rise can be attributed to transmission between the LUMO state and also the lower HOMO state. The qualitative and quantitative agreement with the experimental data of Reed et al. [1] (the dashed curve in Fig. 2(b)) in the voltage range below $2 \mathrm{~V}$ is quite striking: the features in the calculated differential conductance curve appear at nearly the same values of the bias as in the experimental data, and also the magnitude of the calculated conductance is close to that which was observed in the experiment. Above $2 \mathrm{~V}$ the qualitative agreement is less striking, yet the overall magnitude is in quantitative agreement with the measured values. In the non-linear regime, the effects of electro- 
static potential are undoubtedly important and contribute to the shifting of the molecular orbitals. This would lead to shifting of the resonances shown in Fig. 2(a). We have attempted to address qualitatively what this shifting may look like by introducing potential drops at the contacts between the leads and molecules as well as in between the two molecules. We assumed that $75 \%$ of the voltage drops between the two molecules while the other $25 \%$ drops at the metal contacts (this is a simple assumption which may or may not reflect the true potential profile of the system). We found that the resonances do shift somewhat but that the overall magnitude of the resonances does not change appreciably. To obtain more accurate results at the higher biases, more sophisticated approaches which are based on density-functional theory should be used. Other improvements might be to use Monte-Carlo techniques to achieve a proper Boltzmann sampling of the possible configurations of the two molecules.

\section{Spin-valve behavior in a ferromagnetic break- junction bridged by self-assembled monolayers of an organic molecule}

In this section, we explore the transport properties of a break-junction bridged by SAMs of organic molecules where the break-junction is made of ferromagnetic nickel. Ferromagnets bridged by conducting layers are of interest from a device standpoint in part because of the possibility of spin-valve behavior. A spin-valve is characterized by a change in the conductance of the system when the magnetizations of the two ferromagnetic layers switch between parallel and anti-parallel configurations.

To gain some intuitive insight into this behavior, it helps to consider the simplified band structure of ferromagnetic nickel shown schematically in Fig. 3. Nickel is a ferromagnet because it has unequal spin up and spin down populations. The d-band for spin up electrons is completely filled, while for spin down electrons it is partially filled. This gives nickel a net spin which implies a net magnetization even in zero applied magnetic field. Now consider an experiment that measures the
Nickel Band Structure

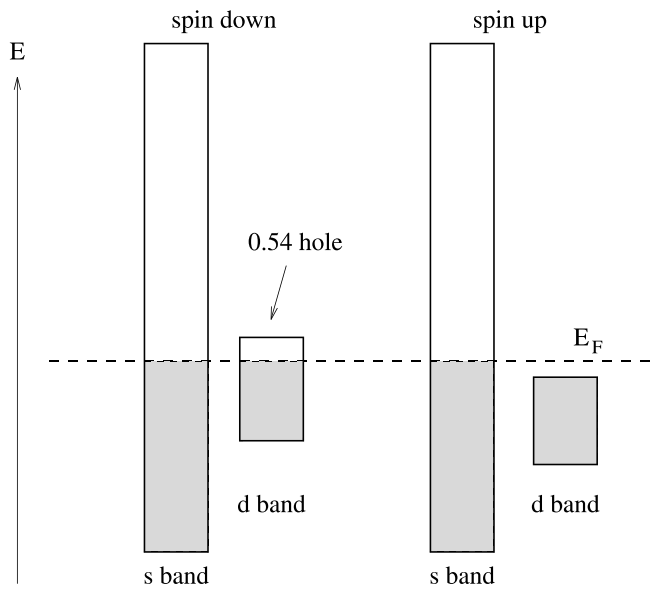

Fig. 3. Simplified schematic of the band structure of ferromagnetic nickel. On the left, the band structure of spin down electrons. The d-band is partly empty. On the right, the band structure for the spin up electrons. Here, the d-band is completely occupied. The $0.54 \mathrm{e}$ hole in the d-band of the spin down electrons gives Nickel a net magnetic moment of $0.54 \mu_{\mathrm{B}}$.

conductance between two nickel layers. In the situation where the two layers have their magnetizations aligned parallel, spin down electrons at the Fermi energy in the d-band of one layer will be transported into both the d-band and the s-band of spin down electrons of the other layer (ignoring spin-flip scattering and assuming that there is a conducting channel between the two layers). Then consider the situation where the magnetizations are aligned anti-parallel. Now the spin down electrons at the Fermi level in the d-band of one layer will be transported into the s-band of the spin up electrons of the other layer, since there is no dband for spin up electrons in the drain at the Fermi energy. This simple argument implies that the band mismatch in the anti-parallel configuration will lead to a different conductance. Hence one might expect a difference in conductance between the two configurations. Non-magnetic metals, thin insulating layers and vacuum tunnel barriers are among the systems that have been used successfully as conduits between ferromagnetic metal layers in such spin-valve experiments. Here we consider the conduit to consist of self-assembled monolayers of organic molecules. 
The structure of the system we consider is similar to that of the system studied in the preceding section but here the nanoscale contacts are of $(100)$ nickel. Again they are in the form of clusters built from $5 \times 5,4 \times 4,3 \times 3$ and $2 \times 2$ layers of atoms. We also make the assumption that each cluster forms a single magnetic domain. We again consider BDT to be the molecule forming the SAM on each tip. We assume that each sulfur atom that binds to a tip is situated 1.7 $\AA$ above a fourfold hollow site of nickel. The tight-binding parameters that we use for nickel's $4 s, 4 p$ and $3 d$ orbitals are taken from [57] for bulk ferromagnetic nickel. There are two sets of parameters, one for spin up electrons and one for spin down electrons. The parameters for the atoms making up the molecules are taken from the standard extended Hückel set [55]. We evaluate the Hamiltonian matrix elements coupling the nickel tips to the molecules using extended Hückel where now the $\mathrm{Ni}$ parameters are the standard ones used in extended Hückel calculations [55]. Thus, we make the assumption that spin down and spin up electrons interact in the same manner with the molecule. The energy scale used in [57] is offset relative to that used in extended Hückel theory. Thus to achieve consistency between the two schemes we added a constant $\epsilon=-17.2 \mathrm{eV}$ to all of the site energies taken from [57] and also adjusted the off-diagonal Hamiltonian matrix elements accordingly; for further details of this procedure see Appendix A. (The shift was calculated by aligning the Fermi energies: $E_{\mathrm{F}}=8.7 \mathrm{eV}$ from Papaconstantopoulos and $E_{\mathrm{F}}=-8.5 \mathrm{eV}$ for bulk $\mathrm{Ni}$ as calculated from the parameters used by extended Hückel.) Again, $6 \times 6$ simple cubic semi-infinite ideal leads were coupled to the last layer of each cluster, with an $\mathrm{Ni}$ s orbital on each site. The coupling between the ideal leads and the tips were evaluated using extended Hückel.

We performed transport calculations for the cases where the magnetizations of the single domain tips are aligned parallel and anti-parallel. The transmission probabilities for these two cases are shown in Fig. 4. Because the dimensionality of the Hamiltonian matrix has grown ninefold over the case where the tips were made of gold,
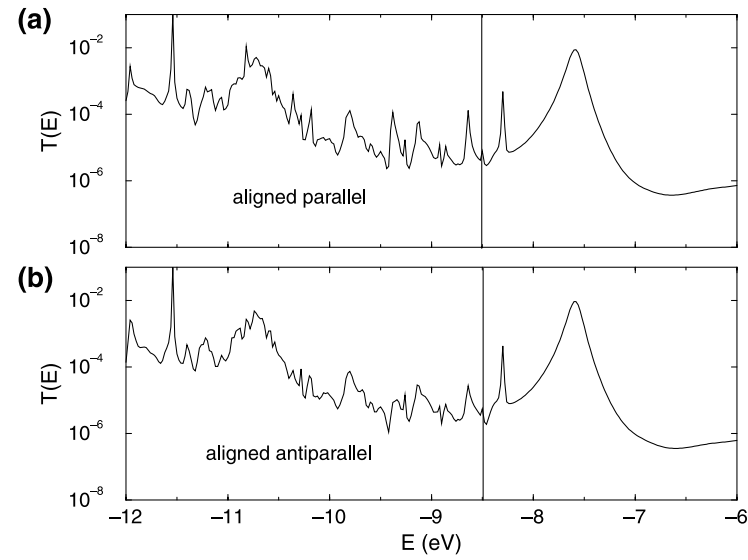

Fig. 4. Transport properties for a single configuration of an overlapping pair of BDT molecules bonded to an Ni breakjunction: (a) total transmission probability for the case where the magnetizations on the left and right leads are aligned parallel; (b) total transmission probability for the case where the magnetizations are aligned anti-parallel. The vertical line indicates the chosen Fermi energy for the nickel leads of $-8.5 \mathrm{eV}$ (see text).

we did not perform averaging over the orientations of the two molecules - what is shown is $T(E)$ for only one particular configuration. (Our purpose here is not so much to make quantitative predictions, but simply to use the semi-empirical approach to gain insight into what might be observed since, as was shown in the preceding section, calculations such as this can make reasonable assessments about the magnitude of transmission and also the hybridization that occurs between the molecules and the metal.) Fig. 4(a) shows the total transmission for the case where the tips' magnetizations are aligned parallel. Two calculations were performed to generate this curve. The first involved calculating $T_{\text {down }}(E)$ for the system where spin down parameters are used on both tips, and then secondly calculating $T_{\text {up }}(E)$ for the case where spin up parameters are used on both tips. The total transmission is then $T(E)=T_{\text {up }}(E)+T_{\text {down }}(E)$. Above $-8.5 \mathrm{eV}$, the dominant channel in Ni is s-type, and hence $T(E)$ displays similar features to those seen for the gold break-junction system: There is a resonance at $-8.2 \mathrm{eV}$ from the LUMO and a broader peak at higher energy from the next LUMO. Below -8.5 
$\mathrm{eV}$, we see the effects of the d-states of $\mathrm{Ni}$. The HOMO and LUMO hybridize with the d-states of Ni near the Fermi energy to form transporting states throughout the nominal HOMO-LUMO gap of the molecule. Fig. 4(b) shows $T(E)$ for the case where the tips are aligned anti-parallel. This curve was calculated by using spin down parameters on one tip and spin up parameters on the other. This yields $T_{\text {down } \rightarrow \text { up }}(E)$ and due to detailed balance the total transmission is $T(E)=2 T_{\text {down } \rightarrow \text { up }}(E)$. Because of the band mismatch, there are some differences between the parallel and anti-parallel configurations. The first resonance below $-8.5 \mathrm{eV}$ is clearly lower in amplitude for the case where the spins are aligned anti-parallel compared to the parallel case. This should manifest itself as a difference in conductance between these two configurations.

In Fig. 5, we show the calculated differential conductance for the break-junction system at room temperature using a Fermi energy of -8.5 $\mathrm{eV}$. The solid line corresponds to the case where the two tips have their magnetizations aligned parallel. One might imagine separating the tips and applying a magnetic field so as to flip the spin on one of the tips. Alternatively, the break junction may be fabricated so that the Ni film on one side of the break junction is thinner than on the other; the magnetization of the thinner film will

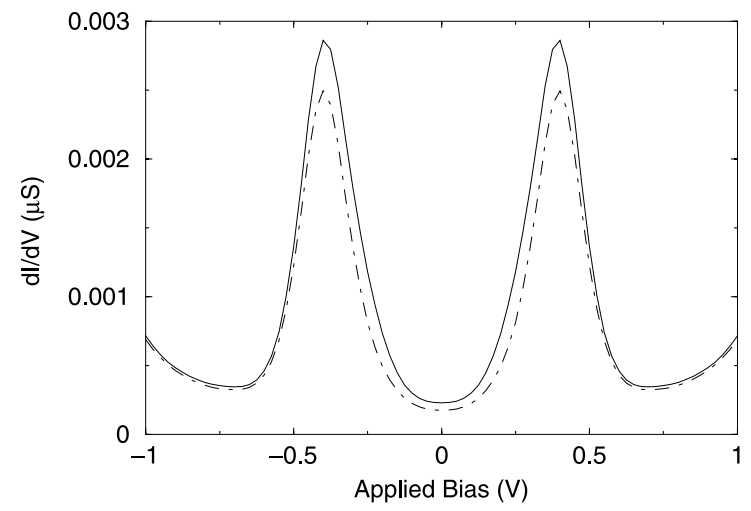

Fig. 5. Calculated differential conductances of the Ni-BDT break-junction system at room temperature using a Fermi energy of $-8.5 \mathrm{eV}$. (Solid line) case where the magnetizations of the two tips are aligned parallel. (Dashed line) case where the magnetizations are aligned anti-parallel. reverse at a lower applied magnetic field than that of the thicker film. The dashed line shows the differential conductance for the situation where the tips are aligned anti-parallel. The difference in conductance is not dramatic, although it should be within the realm of experimental detection. Thus this simple model suggests that spin-valve behavior should observable in a break-junction measurement.

\section{Spin-dependent transport in STM measurements on organic molecules}

In this section, we consider some possible experiments which utilize STM apparatus with an STM tip made from single crystal nickel with a single magnetic domain. Specifically we consider two systems: one with an SAM grown on a substrate of gold and second one where the SAM is grown on nickel. We consider the SAM to be made from benzene-thiol (BT), where the thiol binds to the substrate. The end of this molecule that does not carry the thiol group should not bond chemically to the Ni STM tip, and we will assume that the tip is clean, i.e., free from adsorbed molecules. For the system with the gold substrate, we assume the possibility of detecting any spin-polarized current that may be injected into it from the STM tip. For the case where the substrate is nickel, we are again interested in the possibility of spin-valve behavior.

Fig. 1(b) is a schematic of the set-up that we consider. The substrate is taken to consist of $\left(\begin{array}{lll}1 & 0 & 0\end{array}\right)$ metal, and we model it using a rounded tip consisting of $5 \times 5,4 \times 4,3 \times 3$, and $2 \times 2$ layers of atoms. (As an aside, we found that using a larger approximately cubic cluster to model the substrate resulted in dimensional resonances of the cluster itself being interspersed with the transmission features that concern us here that are due to the molecular wire and its coupling to the metal contacts. Using the rounded tip removes these dimensional resonances from the energy range of interest, and the resonances presented below are robust to changes of the ideal leads, the coupling of leads to the clusters, and disorder.) The tip is also taken to be in the $\left[\begin{array}{ll}1 & 0\end{array}\right]$ ] direction, and consists 
of $5 \times 5,4 \times 4,3 \times 3,2 \times 2$ layers of atoms which are capped with a single atom. The molecule is assumed to be perpendicular to the surface with the sulfur atom situated over a fourfold hollow site on the substrate. For the case where the substrate is gold, we assume a binding distance of $2 \AA$, and when it is nickel, a distance of $1.7 \AA$. We assume that the STM tip is situated over the principal axis of the molecule at a distance of $2 \AA$ from the end hydrogen atom. We use only the 6 s orbital for the gold atoms in the substrate. For the tip, since there is now a single atom forming the end of the tip, we use the full valence orbital set of gold. This is done because of symmetry considerations: The s orbital at the end of the tip is orthogonal to the $\pi$ states of the molecule, whereas the $\mathrm{p}$ and $\mathrm{d}$ states are not. For systems where the molecule is coupled to only a single atom, we have found that including the $p$ and $\mathrm{d}$ orbitals leads to different transmission characteristics from the case where only the s orbital was used; we again take the parameters from extended Hückel.

In the case where both the substrate and STM tip are $\mathrm{Ni}$, we again use Papaconstantopoulos' [57] parameters for both the spin up and spin down bands adjusted for consistency with the molecular extended Hückel parameters as was discussed in Section 4. In the case where the STM tip is Ni and the substrate is gold, it is necessary to address the further complication that although the tip and substrate are of different metals, the Fermi levels (or, in more precise terms, the electro-chemical potentials) of the tip and substrate must be equal at zero bias if the two metals are in electrical contact with each other through the molecular wire. (If their Fermi levels were not aligned at zero bias then an electric current would flow until an equilibrium state were reached in which the electrostatic potential due to the charge transferred between the two metals would align their Fermi levels and the current would cease to flow.) This Fermi level alignment is taken into account in our calculation by applying an equal shift to the site energies and Fermi level of $\mathrm{Ni}$ given in [57], the shift $(-18.7 \mathrm{eV})$ being chosen so as to align the Fermi level of Ni to the Fermi level for gold ( -10 $\mathrm{eV}$ ) obtained using the extended Hückel parameters. Again the off-diagonal matrix elements of the tight-binding Hamiltonian for $\mathrm{Ni}$ are adjusted accordingly. We treat the molecule using extended Hückel. As was done previously for the Ni breakjunction system, the coupling parameters between $\mathrm{Ni}$ and molecule are evaluated using extended Hückel.

Transmission probabilities for the situation where the substrate is gold are shown in Fig. 6. Fig. 6(a) is for the case where the STM tip is made of gold; we include it as a reference for comparison with our results for the more complicated magnetic case where the STM tip is made of nickel. The features seen in $T(E)$ in Fig. 6(a) are qualitatively similar to what was seen for the break-junction system. The resonance at $-8 \mathrm{eV}$ is due to the LUMO of BT. There is an overall rise in transmission below $-10 \mathrm{eV}$ which is due to the hybridized HOMO states.
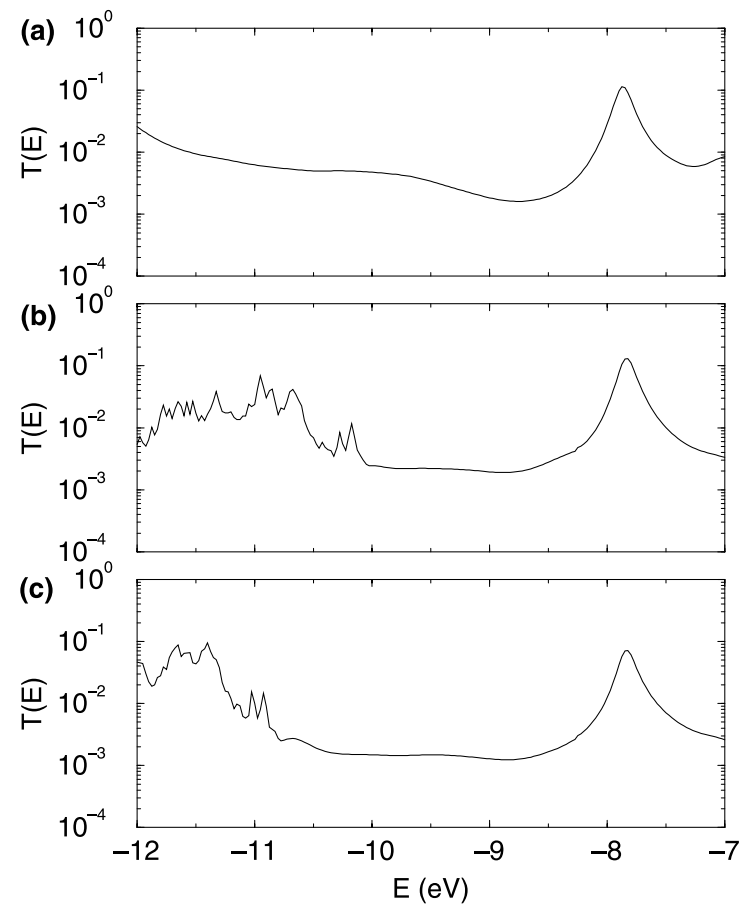

Fig. 6. Transmission probabilities for the STM set-up using a gold substrate and benzene-thiol as the molecular wire: (a) case where the STM is gold; (b) transmission probability for spin down electrons for the Ni tip; (c) transmission probability for spin up electrons for the Ni tip. 
The calculated $T(E)$ for the case where the tip is made of Ni are shown in Figs. 6(b) and (c): (b) shows $T_{\text {down }}(E)$ for the spin down electrons. (c) shows $T_{\text {up }}(E)$ for the spin up electrons. Again, the d-states from the Ni hybridize with the molecular states to provide channels for transport. There are striking differences between the two curves below $-10 \mathrm{eV}$ (the adjusted Fermi energy of the Ni tip). Because the spin down d-band straddles the Fermi energy there are d-state resonances near $-10 \mathrm{eV}$. For the spin up electrons, the d-band begins about $1 \mathrm{eV}$ below this, and so no spin up d-states contribute to transport within this window of energy. Because of this difference, there is a net difference between the number of spin down versus spin up electrons transmitted into the substrate. This generates a non-equilibrium population of spin states in the s-band of the gold substrate. To quantify this difference we have calculated the current due to spin up and spin down electrons in this system as a function of applied bias. This is shown in Fig. 7. The spin down current is greater than the spin up current by a factor of approximately $2-4$. Thus a strong spin polarization of the current should be expected in this system.

The last case that we consider is that where both the tip and substrate are made of nickel.

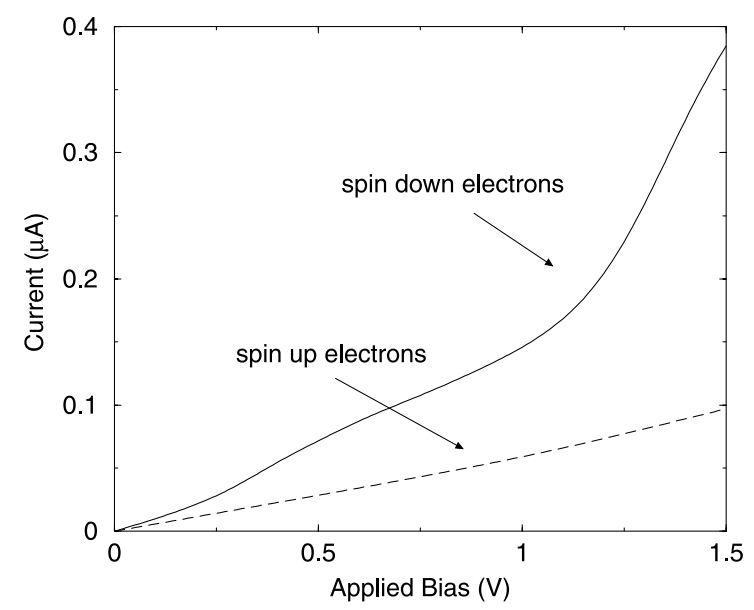

Fig. 7. Calculated current at room temperature as a function of applied bias for the Ni STM tip - BT - Au substrate system. (Solid line) current due to spin down electrons. (Dashed line) current due to spin up electrons.
Here, we are again interested in whether spinvalve behavior should be observable. Since the only metal present in the system is $\mathrm{Ni}$, we revert to the tight binding and Fermi energy parameters that were described in Section 4. The transmission probability for this system where the magnetizations of the substrate and tip are aligned parallel and anti-parallel are shown in Figs. 8(a) and (b), respectively. For the parallel case there is a resonance near $-8.7 \mathrm{eV}$ which is strongly suppressed in when the magnetizations are anti-parallel. This should manifest itself as a noticeable difference in differential conductance. Fig. 9 shows the calculated differential conductance at room temperature, again using a Fermi energy of $-8.5 \mathrm{eV}$ for nickel [58]. The spin-valve effect is now more prominent than that predicted for the breakjunction system where the difference between the strengths of the resonances in the parallel and antiparallel configurations is not as large. The first rise in conductance for the parallel aligned configuration in Fig. 9 is due to transport through the resonance at $-8.7 \mathrm{eV}$. This resonance is much weaker in the anti-aligned configuration, as is the corresponding feature in the conductance in this case. The difference in conductance between the two cases is now a factor of two, which should be readily detectable.
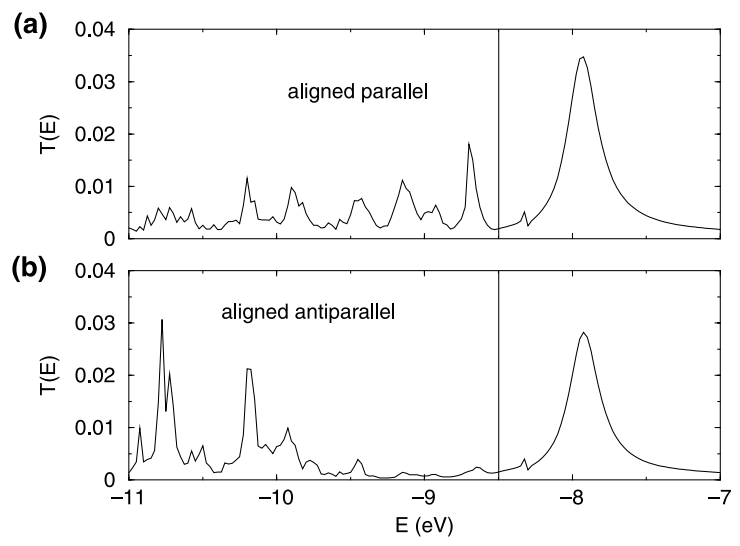

Fig. 8. Transmission probabilities for the case where the substrate is nickel: (a) and (b) total transmission probability for the case where the substrate and tip have their magnetizations aligned parallel and anti-parallel, respectively. 


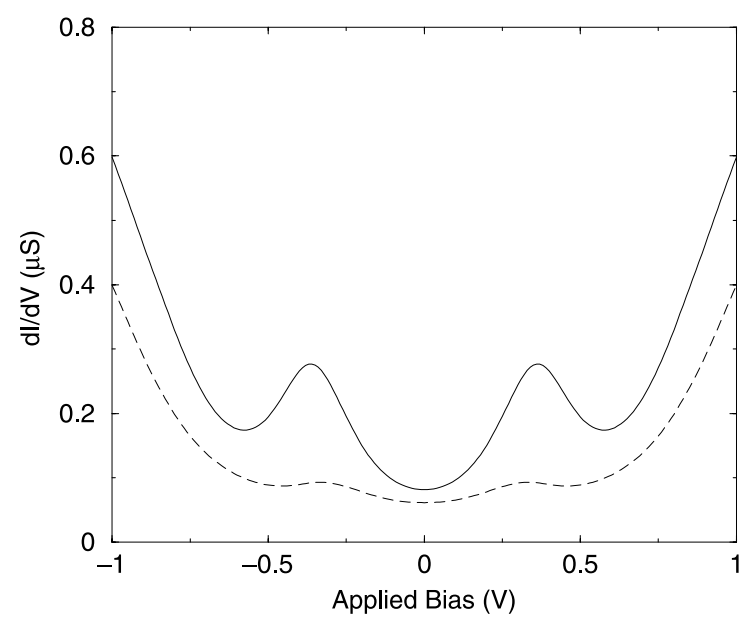

Fig. 9. Calculated differential conductance at room temperature for the Ni STM tip-BT-Ni substrate system, using a Fermi energy of $-8.5 \mathrm{eV}$. (Solid line) STM tip and substrate magnetizations aligned parallel, (dashed line) aligned anti-parallel.

\section{Conclusions}

In conclusion, we have shown that spin-dependent transport should be readily observable in molecular wire systems. First, we gave a summary of a model of electrical conduction through selfassembled molecular mono-layers that bridge metal break-junctions [22]. We assumed that current flows through overlapping molecules each of which is chemically bonded to only one tip. We showed that this model can quantitatively and qualitatively reproduce the experimental data reported by Reed et al. [1] for a non-magnetic system. Using this model, we then proceeded to show that it should be possible to observe spin-valve behavior in a break-junction formed from ferromagnetic nickel and bridged by SAMs of 1,4benzene-dithiol. The spin-valve behavior occurs because of band mismatch between the two ferromagnetic leads, with the molecules providing transporting states between the two leads. The second class of systems we considered was a variety of STM configurations, with the STM tip made from single-crystal and single-domain nickel. We showed that it should be possible to inject a highly spin-polarized current into a gold substrate which has a self-assembled monolayer of benzene-thiol on it. Lastly, we showed that spin-valve behavior should be detectable if the substrate is nickel covered by an SAM of benzene-thiol. We found that the STM set-up produced a larger spin-valve effect than the break-junction system. These calculations show that semi-empirical transport methods can be useful in exploring phenomenology of molecular wires, and yield results that should be helpful in understanding which effects play an important role in governing electron transport.

\section{Acknowledgements}

We would like to thank Ross Hill, Bret Heinrich, and Alistair Rowe for rewarding discussions and helpful suggestions. This work was supported by NSERC and by the Canadian Institute for Advanced Research.

\section{Appendix A}

Here we review how to change the Hamiltonian matrix elements when the energy is rescaled and a constant energy shift is added to the scattering problem for the case where the underlying basis is non-orthogonal. Schrödinger's equation for the scattering problem is $H|\Psi\rangle=E|\Psi\rangle$. We can scale this equation by a factor $c$, and add and subtract a constant energy shift $\epsilon$ yielding

$c H|\Psi\rangle=c E|\Psi\rangle+\epsilon|\Psi\rangle-\epsilon|\Psi\rangle$.

We redefine the scattering energy to be $E^{\prime}=$ $c E-\epsilon$. Using a non-orthogonal basis the above equation becomes a matrix equation,

$\sum_{j}\left(c H_{i j}-\epsilon S_{i j}\right) \Psi_{j}=E^{\prime} \sum_{j} S_{i j} \Psi_{j}$

We can define a new Hamiltonian matrix after the application of a scaling and a constant energy shift by

$H_{i j}^{\prime}=c H_{i j}-\epsilon S_{i j}$.

Thus we see that not only are the diagonal elements changed by the shift, but also the off- 
diagonal elements when the basis is non-orthogonal.

\section{References}

[1] M.A. Reed, C. Zhou, C.J. Muller, T.P. Burgin, J.M. Tour, Science 278 (1997) 252.

[2] S. Datta, W. Tian, S. Hong, R. Reifenberger, J.I. Henderson, C.P. Kubiak, Phys. Rev. Lett. 79 (1997) 2530.

[3] R.M. Metzger, B. Chen, M.P. Cava, Thin Solid Films 327/ 329 (1998) 326.

[4] C.P. Collier, G. Mattersteig, E.W. Wong, Y. Luo, K. Beverly, J. Sampaio, F.M. Raymo, J.F. Stoddart, J.R. Heath, Science 289 (2000) 1172.

[5] J.K. Gimzewski, C. Joachim, Science 283 (1999) 1683.

[6] J. Reichert, R. Ochs, D. Beckman, H.B. Weber, M. Mayor, H.v. Löhneysen, cond-mat/0106219 (2001).

[7] J. Chen, M.A. Reed, A.M. Rawlett, J.M. Tour, Science 286 (1999) 1550.

[8] J.H. Schön, H. Meng, Z. Bao, Science 413 (2001) 2138.

[9] N.D. Lang, P. Avouris, Phys. Rev. Lett. 81 (1998) 3515.

[10] M. Di Ventra, S.T. Pantelides, N.D. Lang, Phys. Rev. Lett. 84 (2000) 979.

[11] J. Taylor, H. Guo, J. Wang, Phys. Rev. B 63 (2001) 121104.

[12] V. Mujica, A.E. Roitberg, M. Ratner, J. Chem. Phys. 112 (2000) 6834.

[13] E. Emberly, G. Kirczenow, Phys. Rev. B 62 (2000) 10451.

[14] E. Emberly, G. Kirczenow, Phys. Rev. B 64 (2001) 125318.

[15] P.S. Damle, A.W. Ghosh, S. Datta, Phys. Rev. B 64 (2001) 201403.

[16] D.S. Kosov, J.C. Greer, Phys. Lett. A 291 (2001) 46.

[17] R. Gutierrez, F. Grossmann, O. Knospe, R. Schmidt, Phys. Rev. A 64 (2001) 013202.

[18] M. Magoga, C. Joachim, Phys. Rev. B 56 (1997) 4722.

[19] S.N. Yaliraki, M. Kemp, M.A. Ratner, Jnl. Am. Chem. Soc. 121 (1999) 3428.

[20] E. Emberly, G. Kirczenow, Phys. Rev. B 58 (1998) 10911.

[21] L.E. Hall, J.R. Reimers, N.S. Hush, K. Silverbrook, J. Chem. Phys. 112 (2000) 1510.

[22] E.G. Emberly, G. Kirczenow, Phys. Rev. Lett. 87 (2001) 269701;

E.G. Emberly, G. Kirczenow, Phys. Rev. B 64 (2001) 235412.

[23] G.A. Prinz, Phys. Today 45 (1995) 58; Science 282 (1998) 1660.

[24] J.M. Kikkawa, Phys. Today 52 (1999) 33.

[25] S. Datta, B. Das, Appl. Phys. Lett. 56 (1990) 665.

[26] R. Meservey, P.M. Tedrow, P. Fulde, Phys. Rev. Lett. 25 (1970) 1270.

[27] M. Julliere, Phys. Lett. 54A (1975) 225.

[28] M. Johnson, R.H. Silsbee, Phys. Rev. Lett. 55 (1985) 1790.

[29] M.N. Baibich, J.M. Broto, A. Fert, F. Nguyen Van Dau, F. Petroff, P. Etienne, G. Creuzet, A. Friederich, J. Chazelas, Phys. Rev. Lett. 61 (1988) 2472.
[30] S.F. Alvarado, P. Renaud, Phys. Rev. Lett. 68 (1992) 1387.

[31] R. Fiederling, M. Keim, G. Reuscher, W. Ossau, G. Schmidt, A. Waag, L. Molenkamp, Nature 402 (1999) 787.

[32] Y. Ohno, D.K. Young, B. Beschoten, F. Matsukura, H. Ohno, D.D. Awschalom, Nature 402 (1999) 790.

[33] G. Schmidt, D. Ferrand, L.W. Molenkamp, A.T. Filip, B.J. van Wees, Phys. Rev. B 62 (2000) R4790.

[34] E.I. Rashba, Phys. Rev. B 62 (2000) 16267.

[35] G. Kirczenow, Phys. Rev. B 63 (2001) 54422.

[36] V.P. LaBella, D.W. Bullock, Z. Ding, C. Emergy, A. Venkatesen, W.F. Oliver, G.J. Salamo, P.M. Thibado, M. Mortazavi, Science 292 (2001) 1518.

[37] H.J. Zhu, M. Ramsteiner, H. Kostial, M. Wassermeier, H.P. Schönherr, K.H. Ploog, Phys. Rev. Lett. 87 (2001) 16601.

[38] K. Tsukagoshi, B.W. Alphenaar, H. Ago, Nature (London) 401 (1999) 572.

[39] H. Mehrez, J. Taylor, H. Guo, J. Wang, C. Roland, Phys. Rev. Lett. 84 (2000) 2682.

[40] E.G. Petrov, I.S. Tolokh, V. May, Phys. Rev. Lett. 79 (1997) 4006;

E.G. Petrov, I.S. Tolokh, V. May, J. Chem. Phys. 108 (1998) 4386;

E.G. Petrov, I.S. Tolokh, V. May, J. Chem. Phys. 109 (1998).

[41] R. Landauer, IBM J. Res. Dev. 1 (1957) 223; Phys. Lett. 85A (1981) 91.

[42] For a comprehensive review of Landauer-Büttiker theory see S. Datta, Electronic Transport in Mesoscopic Systems, Cambridge University Press, Cambridge, 1995.

[43] Extended Hückel calculations of transport through BDT/ $\mathrm{Au}$ systems have also been reported by S. Datta and collaborators (unpublished).

[44] E. Emberly, G. Kirczenow, Phys. Rev. B 60 (1999) 6028.

[45] In certain semiconductor systems it is expected that spinorbit coupling should give rise to interesting spin transport phenomena [25,46] through the Rashba mechanism [47], however, in this work we are concerned with small molecules consisting of relatively light atoms that are not expected to exhibit pronounced spin-orbit-related transport effects.

[46] F. Mireles, G. Kirczenow, Phys. Rev. B 64 (2001) 24426.

[47] Y.A. Bychkov, E.I. Rashba, J. Phys. C 17 (1984) 6039.

[48] S.P. McGlynn, L.G. Vanquickenborne, M. Kinoshita, D.G. Carrol, Introduction to Applied Quantum Chemistry, Holt, Reinhart and Winston Inc., New York, 1972 (see Chapter 3).

[49] E.G. Emberly, G. Kirczenow, Phys. Rev. Lett. 81 (1998) 5205.

[50] E.G. Emberly, G. Kirczenow, J. Phys. Condens. Mat. 11 (1999) 6911.

[51] S.N. Yaliraki, M.A. Ratner, J. Chem. Phys. 109 (1998) 5036.

[52] N.D. Lang, Ph. Avouris, Phys. Rev. B 62 (2000) 7325.

[53] H.H. Jung, Y.D. Won, S. Shin, K. Kim, Langmuir 15 (1999) 1147. 
[54] H. Sellers, A. Ulman, Y. Schnidman, J.E. Eilers, J. Am. Chem. Soc. 115 (1993) 9389.

[55] S. Alvarez, Table of Parameters for Extended Hückel Calculations, 1985.

[56] We found that the transmission probability was larger for the energetically unfavorable configuration where the two molecules are aligned parallel compared to the more favorable perpendicular alignment.

[57] D.A. Papaconstantopoulos, Handbook of the Band Structure of Elemental Solids, Plenum Press, New York, 1986.
[58] We have assumed symmetric drops in potential at the two metal-molecule interfaces in the Landauer formula, and have not calculated $T(E)$ at higher applied biases. However, the system is asymmetrically coupled and therefore some asymmetry in $\mathrm{d} I / \mathrm{d} V$ may be expected to be observed experimentally as the STM tip is retracted from the molecule and the asymmetry between the couplings at the two molecule-metal interfaces becomes more pronounced. 